Supporting Information

\title{
Fully Biomass-Based Hybrid Hydrogel for Efficient Solar Desalination with Salt Self-Cleaning Property
}

Xiaojie Liu, Yanpei Tian, Yanzi Wu, Fangqi Chen, Ying Mu, Marilyn L. Minus, and Yi Zheng*

Department of Mechanical and Industrial Engineering, Northeastern University, Boston, MA 02115, USA.

E-mail: y.zheng@ northeastern.edu 

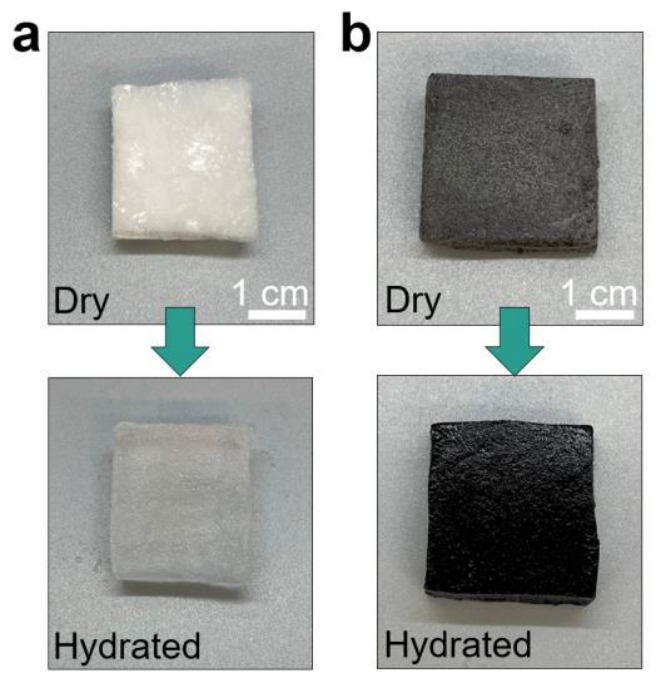

Figure S1. (a) Photographs of pure GG-based aligned porous structure at dry (top) and hydrated (bottom) states, respectively. (b) GG/CI-based aligned porous structure at dry (top) and hydrated (bottom) states, respectively. 

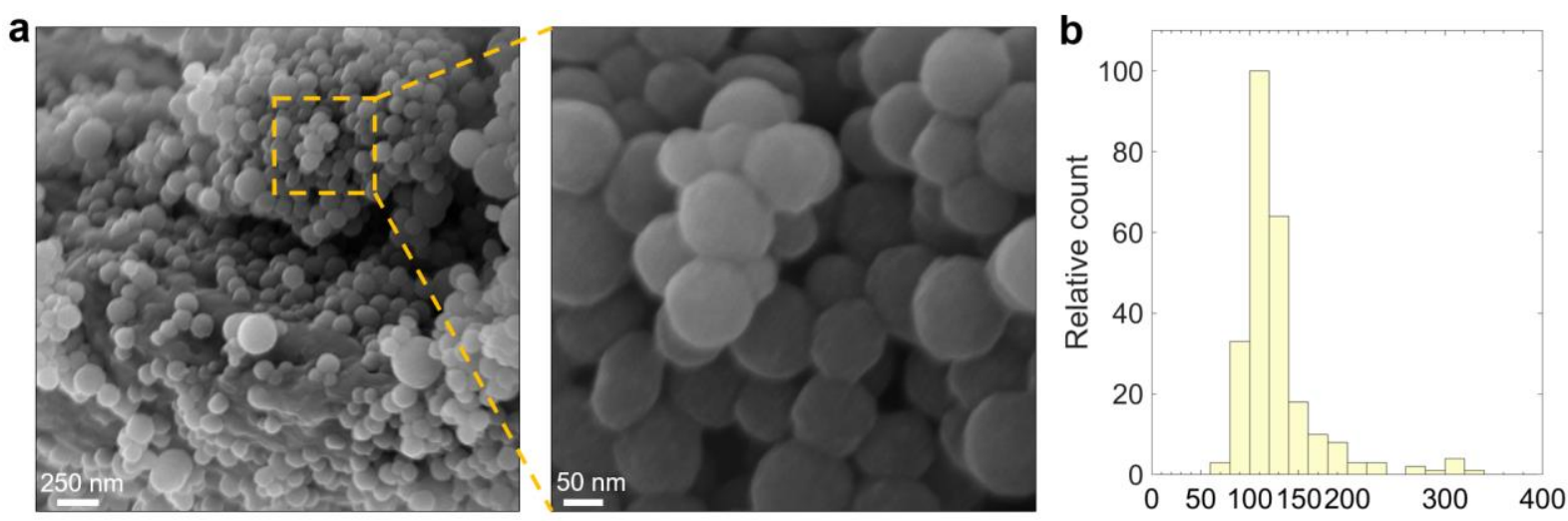

Figure S2. (a) Scanning electron microscopy (SEM) images of cuttlefish ink (CI) powders in different magnifications. (b) The statistical analyses of the diameters of CI powders. 

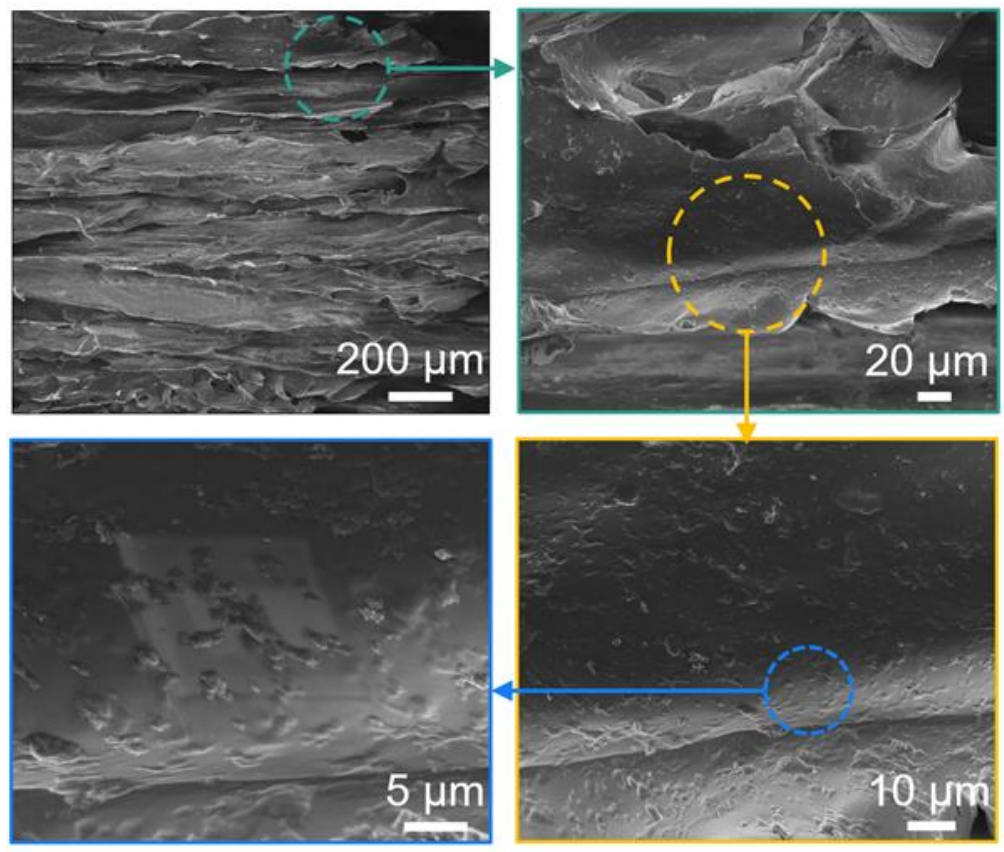

Figure S3. SEM images showing the distribution of CI powder in the longitudinal section of GG/CI aligned porous structure with different magnifications. 

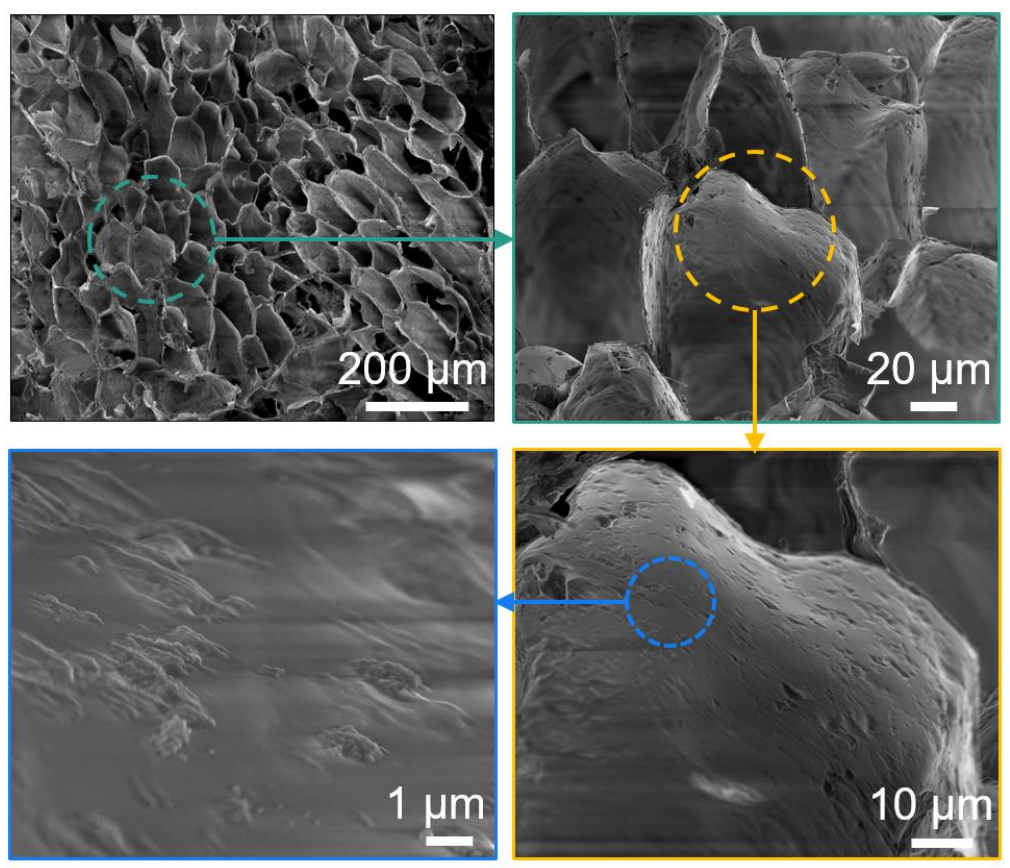

Figure S4. SEM images showing the distribution of CI powder in the transverse section of GG/CI aligned porous structure with different magnifications.

SEM images of the GG/CI aligned porous structures with longitudinal section and transverse section are provided in Figure S3 and Figure S4, respectively, with different magnifications. It is shown that the there is no aggregation of CI powder in the out surface of the GG hydrogel contributing to the pre-dispersion of CI powder into DI water. There are no large CI powder clusters with a size over $10 \mu \mathrm{m}$ existing within walls of GG hydrogel. The diameter of most CI powder clusters is below $1 \mu \mathrm{m}$. 


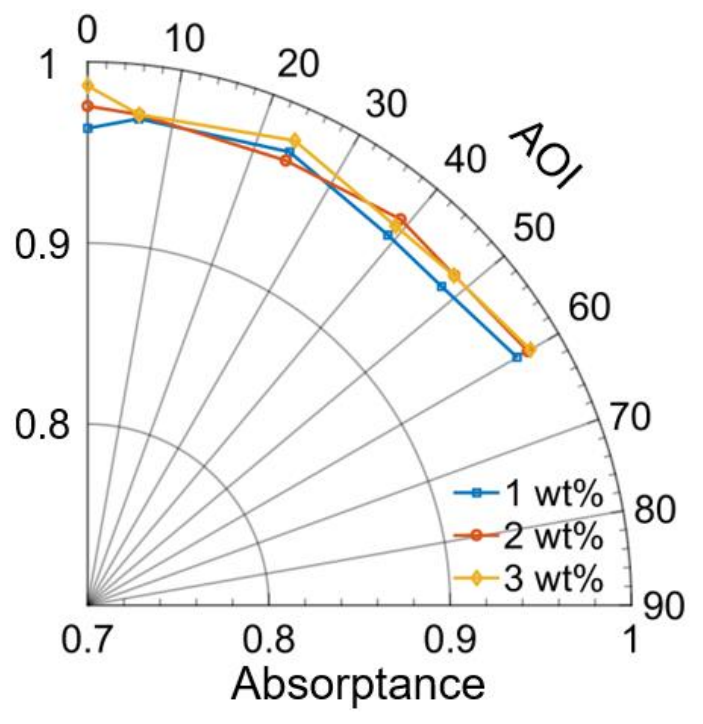

Figure S5. The average solar absorptance of the GG/CI hydrogels across different angles of incidence from $0^{\circ}$ to $60^{\circ}$ in the hydrated state. 


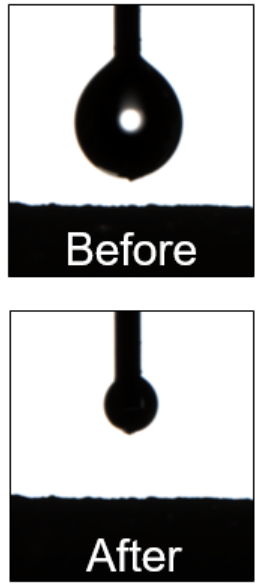

Figure S6. Dynamic contact angle of the $2 \mathrm{wt} \%$ GG/CI hydrogel. 


\section{Saturation water absorption ratio measurement}

The weight of dry hydrogel $\mathrm{m}_{0}$ is measured right after taking it out from the freeze-drying machine. The weight of hydrated hydrogel $\mathrm{m}$ is measured as follows: The crosslinking process of GG/CI hydrogel is performed by immersing the $\mathrm{GG} / \mathrm{CI}$ cryogel into $0.03 \mathrm{wt} \% \mathrm{CaCl}_{2}$ solution under mild shaking for $30 \mathrm{~min}$. Then the GG/CI hydrogel is washed several times with deionized (DI) water to remove the residual $\mathrm{CaCl}_{2}$ solution. After that, the GG/CI hydrogel is placed into DI water overnight to reach fully swelling state. The hydrogel is taken out using a strainer, and the excess water on the outer surface is wiped off with a filter paper, and then the mass of hydrated hydrogel is measured. 


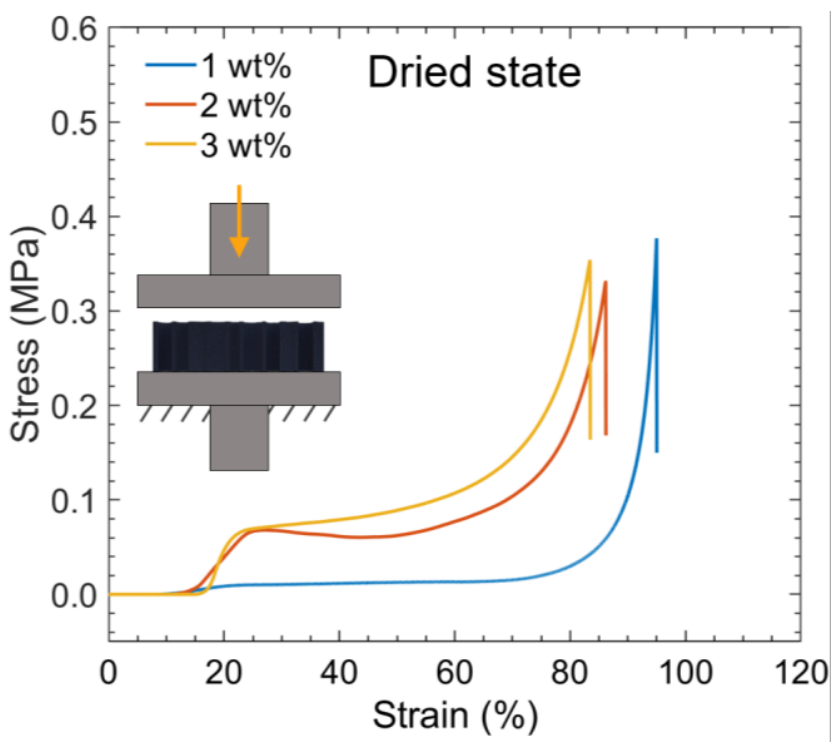

Figure S7. Compressive stress-strain curves of the GG/CI hydrogels with $1 \mathrm{wt} \%, 2 \mathrm{wt} \%$, and 3 $\mathrm{wt} \%$ concentrations in dried state. The inset schematically displays the compression test setup. The GG/CI hydrogel samples are placed on the lower plate and compressed by the upper plate at a compression rate of $3 \mathrm{~mm} \mathrm{~min}$. The compression direction is parallel to the vertical microchannels in the hydrogels. 

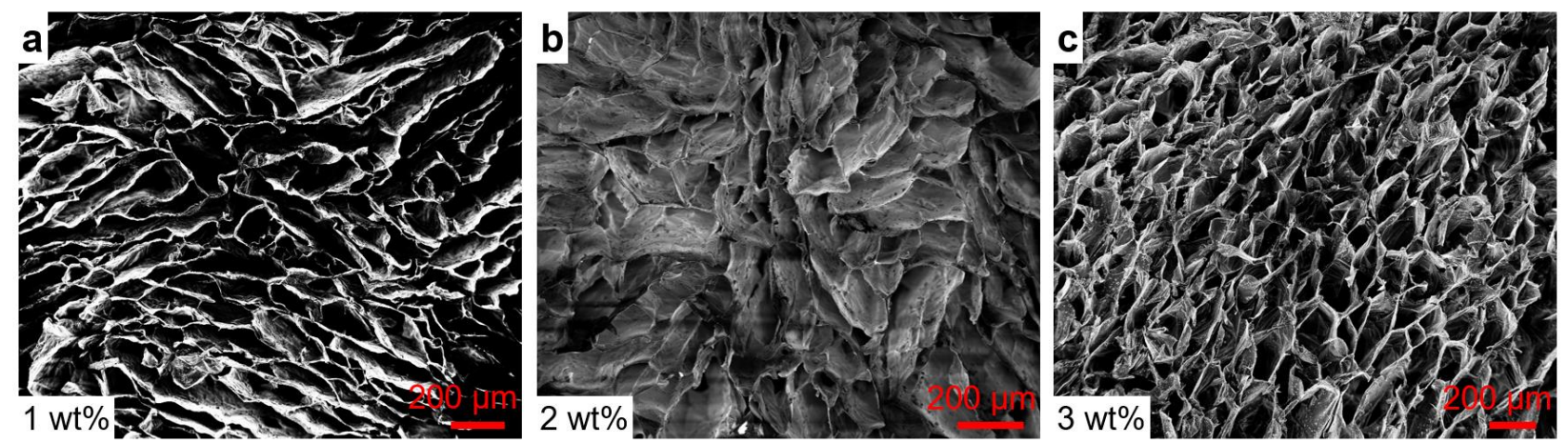

Figure S8. SEM images of the transverse section of GG/CI aligned porous structures with different concentrations of (a) $1 \mathrm{wt} \%$, (b) $2 \mathrm{wt} \%$, and (c) $3 \mathrm{wt} \%$, respectively.

The compressive stress-strain tests are performed on GG/CI hydrogels using a RSA-G2 Solids Analyzer (TA Instruments) at room temperature $\left(21^{\circ} \mathrm{C}\right)$. The GG/CI hydrogel samples are placed on the lower plate and compressed by the upper plate at a compression rate of $3 \mathrm{~mm} \mathrm{~min}^{-1}$. The compression direction is parallel to the vertical microchannels in the hydrogels (Figure S7, inset). For both the dried and hydrated states of GG/CI hydrogel, their fracture stress is all above 0.33 $\mathrm{MPa}$, indicating that they possess a good mechanical strength for the real-life applications. Compared with the $2 \mathrm{wt} \%$ and $3 \mathrm{wt} \%$ GG/CI hydrogel in dried state, the $1 \mathrm{wt} \%$ GG/CI hydrogel displays few stress changes when the compressive strain is smaller than $70 \%$ since it is too soft with a substantial proportion of void volume inside the aligned porous structure. This is because the porosity of $1 \mathrm{wt} \% \mathrm{GG} / \mathrm{CI}$ hydrogel in dry state is higher than those of $2 \mathrm{wt} \%$ and $3 \mathrm{wt} \% \mathrm{GG} / \mathrm{CI}$ hydrogels. It can also be reflected from the SEM images that $1 \mathrm{wt} \%$ porous structure owns a larger pore size (Figure S8). The thermal conductivity of $1 \mathrm{wt} \% \mathrm{GG} / \mathrm{CI}$ hydrogel in dry state is only 0.035 $\mathrm{W} \mathrm{m} \mathrm{K}^{-1} \mathrm{~K}^{-1}$ which is close to that of the air $\left(0.026 \mathrm{~W} \mathrm{~m}^{-1} \mathrm{~K}^{-1}\right)$, indicating the high porosity of $1 \mathrm{wt} \%$ GG/CI hydrogel. For the hydrated hydrogel, all of their fracture stress is higher than $0.35 \mathrm{MPa}$ and their fracture strain is all greater than 92\%, which demonstrates their good mechanical deformability and illustrates a good flexibility for industrial applications. 

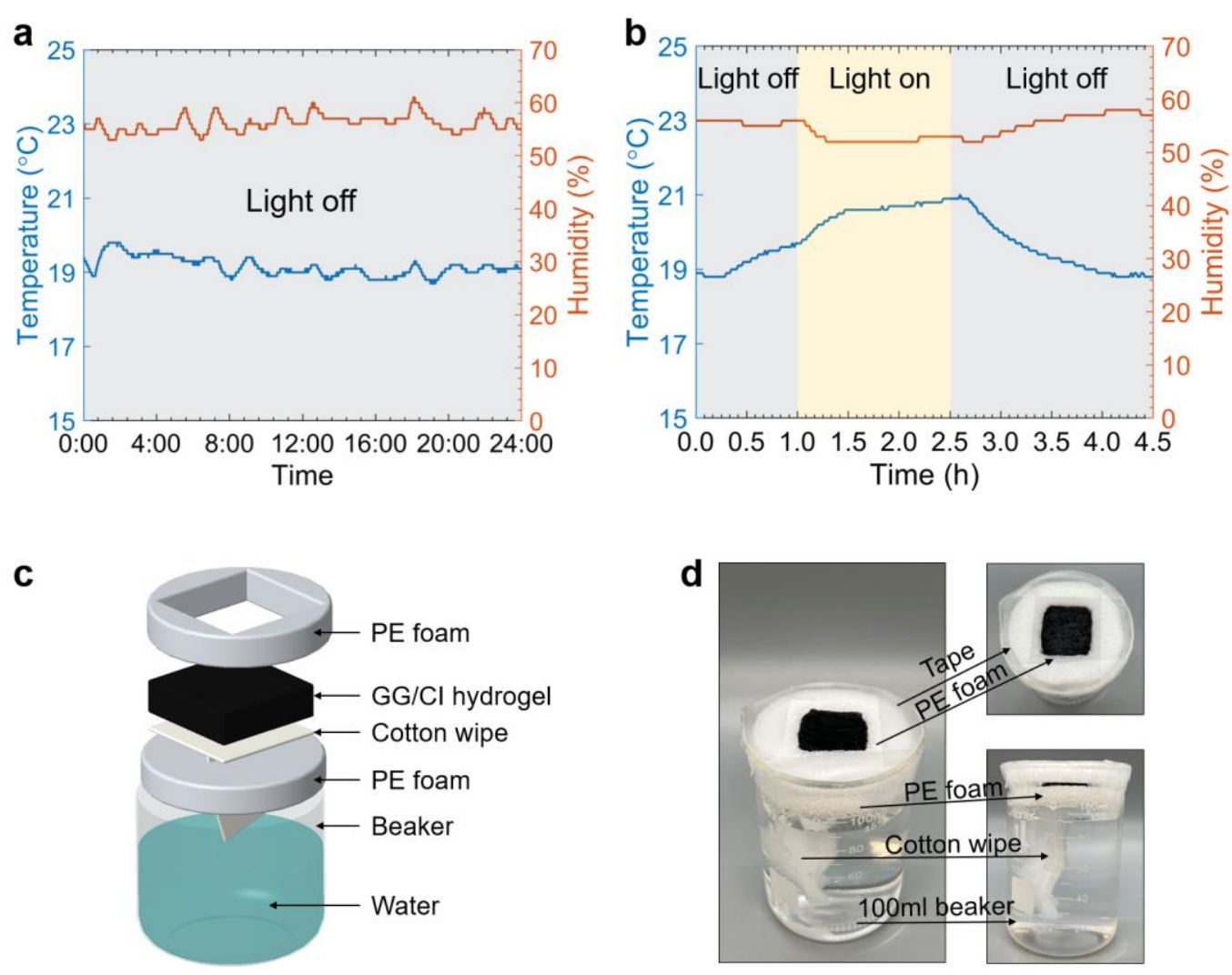

Figure S9. (a) Temperature and relative humidity of the darkroom without solar irradiation over $24 \mathrm{~h}$. (b) Temperature and relative humidity variations of the darkroom during a $1.5 \mathrm{~h}$ water evaporation tests. (c) Schematic illustrating the components of the evaporation device with the GG/CI hydrogel used in this work. (d) Photographs of the evaporation device.

All of tests about the evaporation performance are conducted in a darkroom with the dimension of $3 \mathrm{~m} \times 3 \mathrm{~m} \times 2.5 \mathrm{~m}$. An indoor temperature and humidity sensor located $2 \mathrm{~m}$ away from the solar simulator is used to record the environmental variations of the darkroom. Figure S9a shows the temperature and humidity variations within one day without solar desalination running. The temperature fluctuates around $19.5^{\circ} \mathrm{C}$ and the humidity keeps at around $55 \%$, which are controlled by the central $\mathrm{AC}$ system of the building. The variations of temperature and humidity of the darkroom during a $1.5 \mathrm{~h}$ water evaporation test is shown in Figure S9b. The temperature and humidity are recorded $1 \mathrm{~h}$ before the test and continuously monitored for $2 \mathrm{~h}$ after the experiment finished. The ambient temperature increased from 19 to $20.8^{\circ} \mathrm{C}$ and the humidity decreased from 57 to $52 \%$ after the solar simulator is on. After the test, the temperature and humidity went back to normal. 
As illustrated in Figures S9c and S9d, the GG/CI evaporation device is composed of a $100 \mathrm{ml}$ glass beaker as a water container, a cotton wipe as a water path, a polyethylene (PE) foam as a thermal insulator, and GG/CI hydrogel evaporator. The glass beaker has an inner diameter of $47 \mathrm{~mm}$ and the cotton wipe is $4 \mathrm{~cm}$ in length (immersing in water), $2 \mathrm{~cm}$ in width, and $1 \mathrm{~mm}$ in thickness. The PE foam has a slot in center with a dimension of $2 \mathrm{~cm}$ in length and $2 \mathrm{~mm}$ in width for the cotton wipe to go through. The cotton wipe under the GG/CI hydrogel has a dimension of $2 \mathrm{~cm}$ by $2 \mathrm{~cm}$. The dimension of the GG/CI hydrogel evaporator is $20 \times 20 \times 7 \mathrm{~mm}$. The PE foam on the top layer with the same thickness as the hydrogel is cut off a square to fit for the GG/CI hydrogel evaporator. 

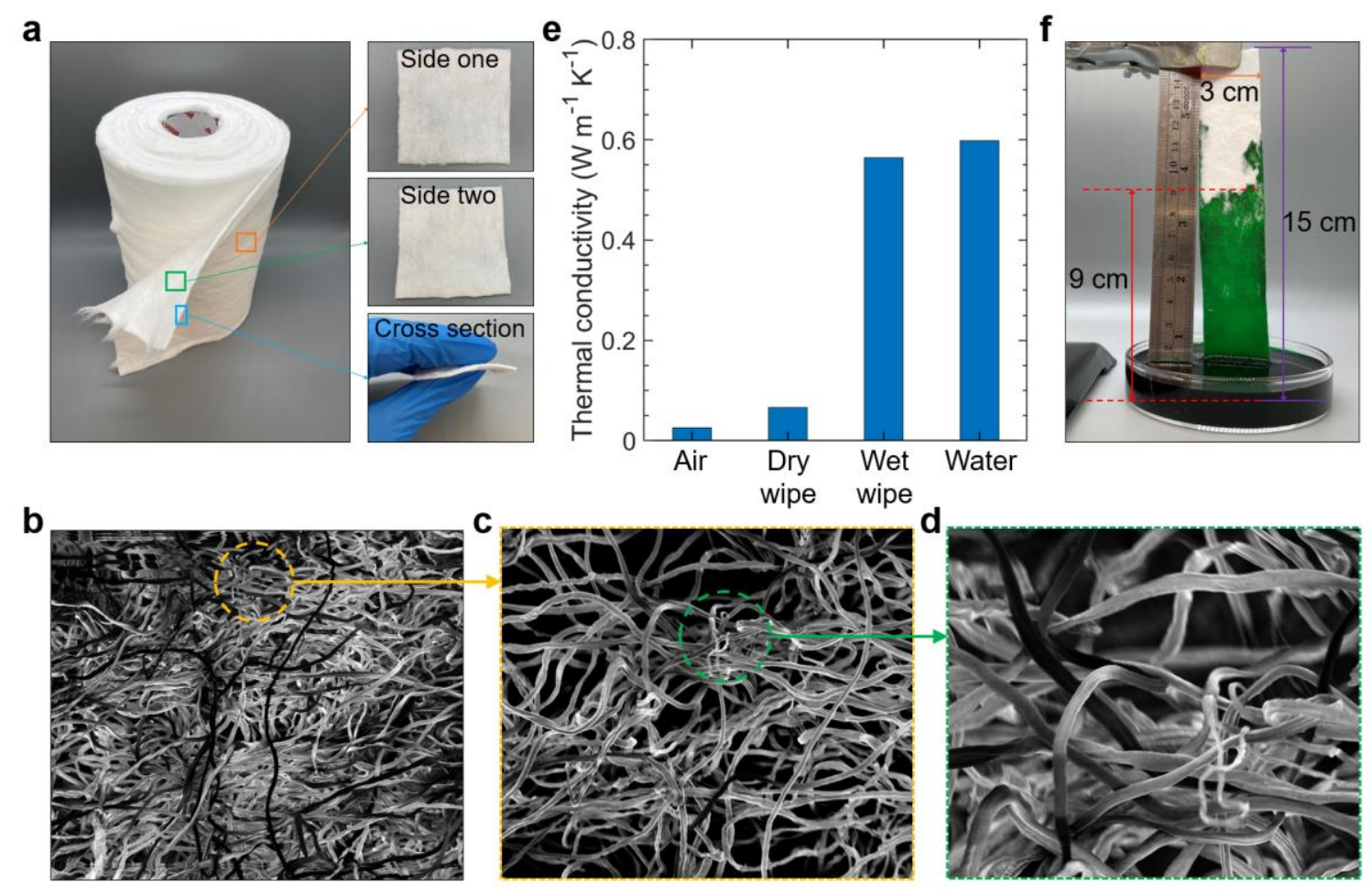

Figure S10. (a) Photographs showing the surfaces and cross-section of the cotton wipe. (b-d) SEM images illustrating the microscale porous structure of the cotton wipe. (e) Thermal conductivities of air, dry cotton wipe, wet cotton wipe, and pure water, respectively. (f) Photograph elucidating the excellent water diffusivity of cotton wipe strip in the vertical direction.

The reason that we select the cotton wipe as the water transportation path is that its porous structures offer excellent water transportation capability and this structures stores sufficient water for the hydrogel evaporator to pump and evaporate. The cotton wipe that was used as the water transportation path is $100 \%$ pure cotton non-woven fabric (Figure S10a). It is soft due to its porous structure formed by randomly arranged cotton fibers, as shown in Figure S10b-d at different magnifications. As shown in Figure S10e, when the cotton wipe is in a dry state, it owns thermal conductivity of $66 \mathrm{~mW} \mathrm{~m}^{-1} \mathrm{~K}^{-1}$ that is at the same order of magnitude of air $\left(26 \mathrm{~mW} \mathrm{~m}^{-1} \mathrm{~K}^{-1}\right)$. This demonstrates that the cotton fibers of cotton wipe form porous structures possessing a large amount of void volume. The porous structure can efficiently absorb water and transfer water to the hydrogel evaporator. The water absorption ratio of the cotton wipe is 14.5 (the weight of the cotton wipe after it fully absorbs water over its dry weight). This is further validated by the thermal conductivity of the wet cotton wipe after it is in a wet state: its thermal conductivity increases to $564.1 \mathrm{~mW} \mathrm{~m}^{-1} \mathrm{~K}^{-1}$. Note that the thermal conductivity of the cotton wipe which fully absorbs water is still smaller than that of pure water $\left(598 \mathrm{~mW} \mathrm{~m}^{-1} \mathrm{~K}^{-1}\right)$, indicating that the cotton wipe also helps to reduce the conductive heat loss compared with the water supply method of direct contact. Figure S10f illustrates the excellent diffusivity of the cotton wipe in the vertical direction. Within 15 minutes, the green-dyed water climbs to $9 \mathrm{~cm}$ above the bulk water surface, and a small amount of the dyed water even goes up to $12 \mathrm{~cm}$ above the bulk water surface. The quick and excellent 
water diffusivity of the cotton wipe ensures the water supply to the hydrogel evaporator and reduces the water contact area between the hydrogel evaporator and the underlying bulk water. 

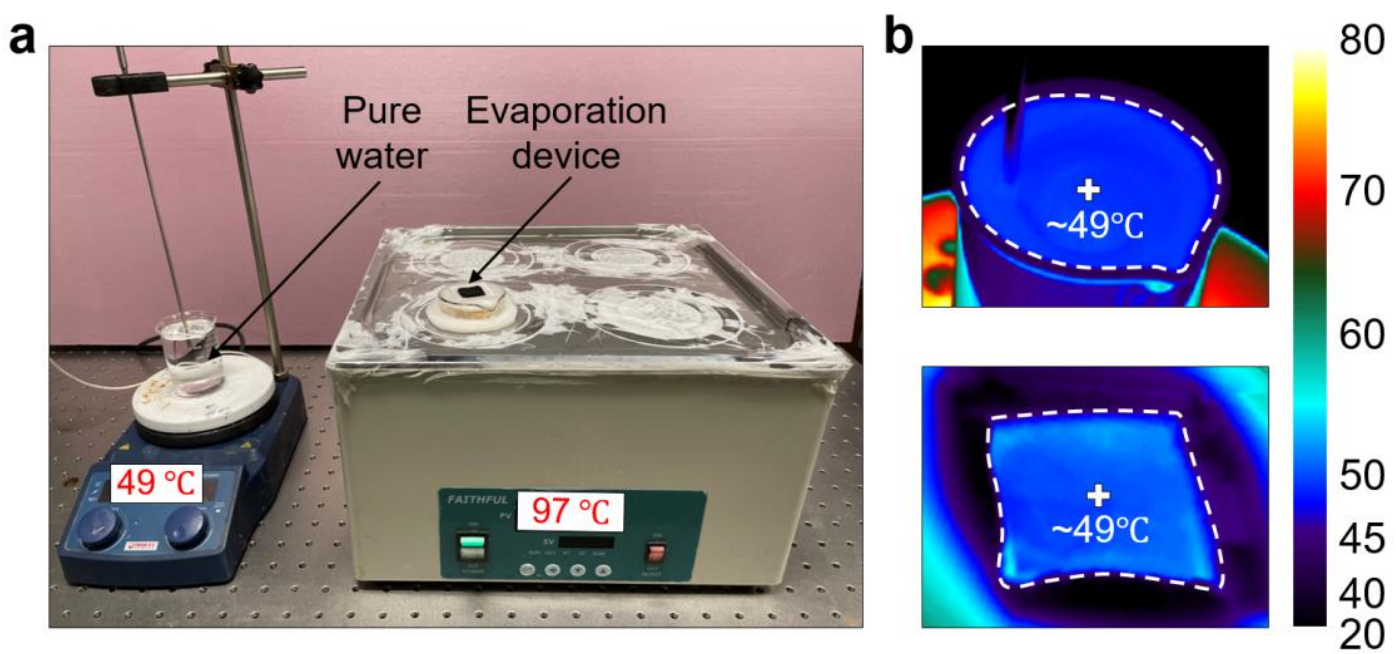

Figure S11. (a) A photograph of the experimental setup for an evaporation rate test under a nonlight condition (dark environment) with the surface temperature of $49^{\circ} \mathrm{C}$. (b) Thermal infrared images displaying the surface temperature distributions of pure water (top) and GG/CI hydrogel (bottom) at the stable states, respectively. The white dashed enclosures showing the evaporation surfaces of pure water and hydrogel. The unit of temperature scale bar is ${ }^{\circ} \mathrm{C}$.

Considering the water evaporation enthalpy is temperature-dependent, the equivalent evaporation enthalpy of water in the GG/CI hydrogel is evaluated under a non-light condition with the evaporation surface temperature of $48^{\circ} \mathrm{C}$. Experimental setup for this dark environment is shown in Figure S7a. The evaporation device with the GG/CI hydrogel is placed into a water bath which stabilizes at $97^{\circ} \mathrm{C}$ to enable the temperature of GG/CI hydrogel surface to be $49^{\circ} \mathrm{C}$. The outer surface of the beaker was surrounded by tapes and foams to seal the gap between the beaker and the holding port of the water bath. Simultaneously, in order to make the GG/CI hydrogel the only outlet for vapor to diffuse out, the small gaps between the opening of glass beaker and the inner $\mathrm{PE}$ foam are also sealed with tape. While the temperature of the pure water surface is kept at $49^{\circ} \mathrm{C}$ using a temperature-controlled hot plate. These surface temperature distributions of the CI/CS hydrogel and pure water are monitored by a thermal camera. After the surface temperatures of the $\mathrm{CI} / \mathrm{CS}$ hydrogel and the pure water are heated and stabilized at $49^{\circ} \mathrm{C}$ for $10 \mathrm{~min}$ (Figure S7b), the initial masses of the evaporation device with GG/CI hydrogel and the pure water with a beaker are weighted. After 2 hours in dark environment, the final masses are recorded. This experiment is repeated 2 times and the mass changes of water for the evaporation device and the pure water with a beaker are as listed in Table S1 below. 
Table S1. The average evaporation rates of water for evaporation device with GG/CI hydrogel and pure water in different dark environment tests.

\begin{tabular}{|c|c|c|c|}
\hline Test & $\begin{array}{c}\text { Evaporation rate of } \\
\text { pure water } \\
\left(\mathrm{kg} \mathrm{m}^{-2} \mathrm{~h}^{-1}\right)\end{array}$ & $\begin{array}{c}\text { Evaporation rate of GG/CI } \\
\text { evaporation device } \\
\left(\mathrm{kg} \mathrm{m}^{-2} \mathrm{~h}^{-1}\right)\end{array}$ & $\begin{array}{c}\text { Evaporation rate ratio } \\
\text { (pure water/GG/CI evaporator) }\end{array}$ \\
\hline 1 & 1.292 & 3.190 & 0.405 \\
\hline 2 & 1.661 & 3.792 & 0.428 \\
\hline
\end{tabular}

The vaporization enthalpy $h_{v a p}$ for water in the GG/CI hydrogel can be estimated using the following formula:

$$
h_{v a p}=\frac{\dot{m}_{w a t e r}}{\dot{m}_{G G / C I}} h_{f g}^{w}
$$

where $\dot{m}_{\text {water }}$ and $\dot{m}_{G G / C I}$ are the evaporation rates of pure water and evaporation device with GG/CI hydrogel in dark environment, respectively. $h_{f g}^{w}$ is the vaporization enthalpy of pure water at $49^{\circ} \mathrm{C}(2384.3$ $\mathrm{kJ} \mathrm{kg}^{-1}$ ). Accordingly, the equivalent vaporization enthalpy $h_{v a p}$ is

$$
\begin{aligned}
& h_{\text {vap }}=0.405 \times 2384.3=965.641 \mathrm{~kJ} \mathrm{~kg}^{-1} \quad \text { for test } 1 \\
& h_{\text {vap }}=0.428 \times 2384.3=1020.480 \mathrm{~kJ} \mathrm{~kg}^{-1} \quad \text { for test } 2
\end{aligned}
$$

Besides, a differential scanning calorimetry (PerkinElmer DSC 7) is also applied to measure the vaporization enthalpies of pure water and water in the GG/CI hydrogel with a thermal ramp rate of $5{ }^{\circ} \mathrm{C}$ $\min ^{-1}$. Figure S8 shows the change of heat flow signal as a function of temperature. The measured vaporization enthalpy of pure water $(7.9 \mathrm{mg})$ is obtained as $2,479.9 \mathrm{~J} \mathrm{~g}^{-1}$, and the vaporization enthalpy of water (10 mg) in the GG/CI hydrogel (10.3 mg of total mass) is calculated as $1490.11 \mathrm{~J} \mathrm{~g}^{-1}$, which is lower than that of pure water. Furthermore, the value of vaporization enthalpy of water in the GG/CI hydrogel which is obtained via a DSC curve is higher than that in dark environment tests, since the DSC test runs a full dehydration process while the dark environment test presents a slightly dehydration process. 


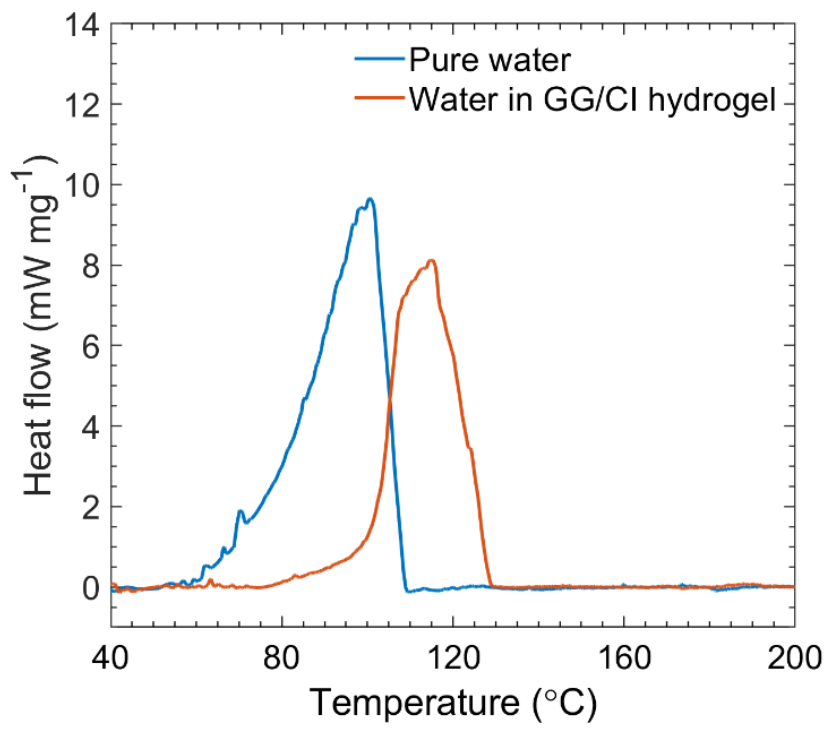

Figure S12. DSC curves of pure water and water in the GG/CI hydrogel. 

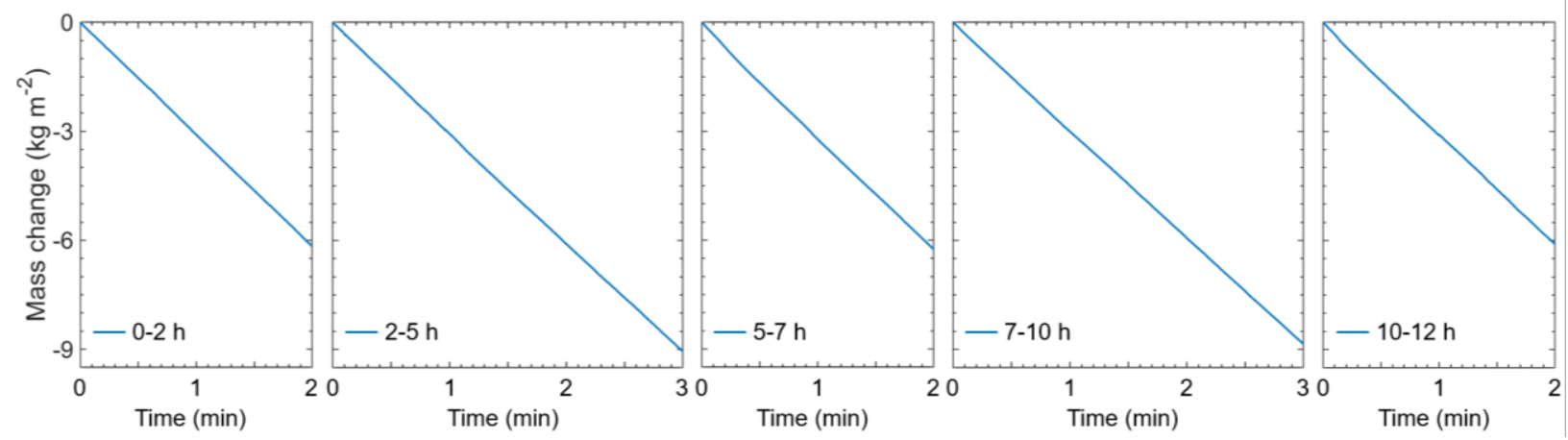

Figure S13. Mass changes of a GG/CI hydrogel evaporator for a $12 \mathrm{~h}$ test under one sun irradiation. To evaluate the stability of solar-driven desalination for GG/CI hydrogel evaporator, a $12 \mathrm{~h}$ duration test was conducted under one sun illumination with $3.5 \mathrm{wt} \% \mathrm{NaCl}$ solution. However, to eliminate the thermal effect of bulk water, which is receiving thermal energy from conductive heat loss from GG/CI hydrogel, bulk water was replaced every two or three hours with the new one at room temperature. The mass changes of water is recorded when the evaporator was at stable state. 

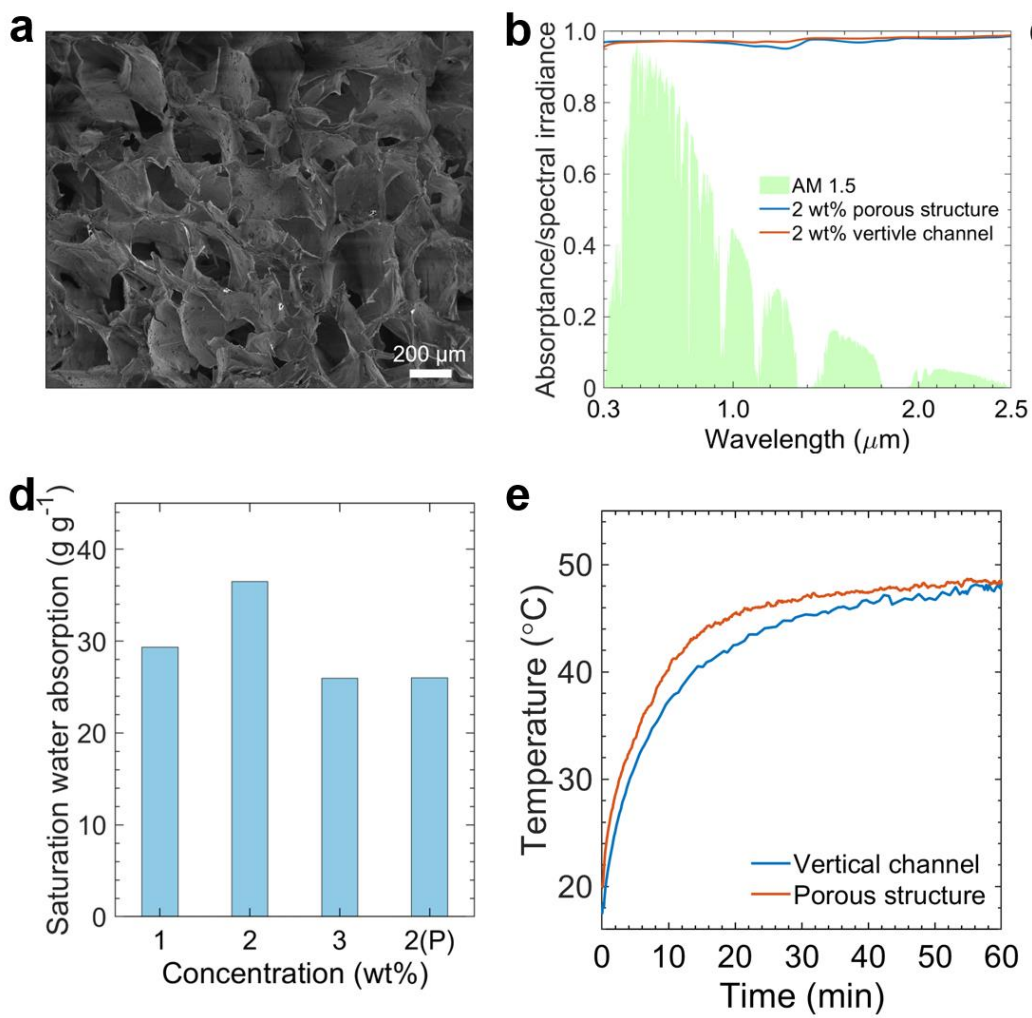
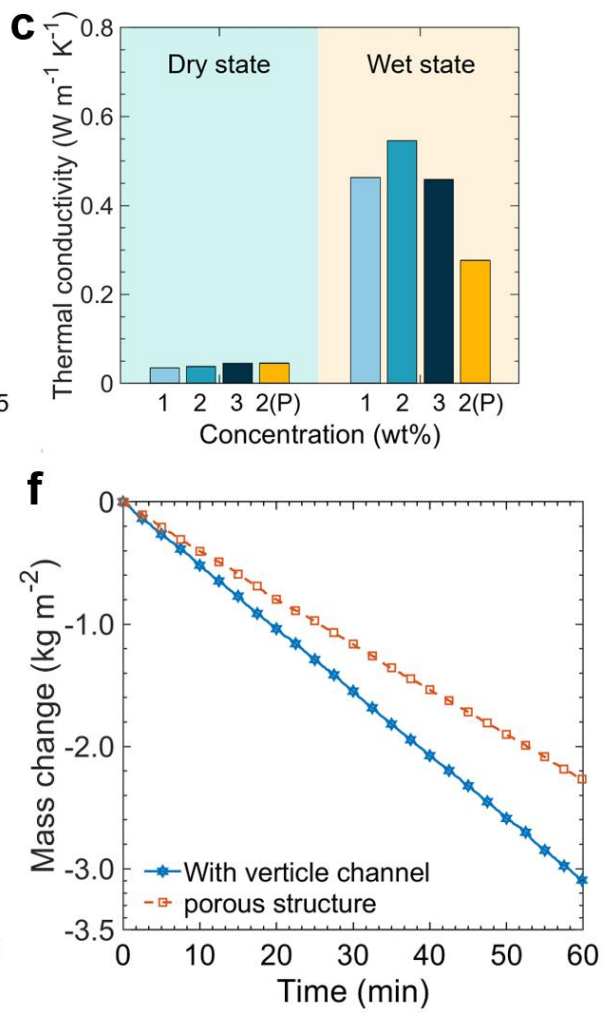

Figure S14. (a) SEM image of the cross-section of GG/CI porous structure with concentration of $2 \mathrm{wt} \%$. The GG/CI hydrogel evaporator with randomly porous structures is fabricated by freeze the $2 \mathrm{wt} \% \mathrm{GG} / \mathrm{CI}$ hydrogel at a $-30^{\circ} \mathrm{C}$ freezer and followed by a freeze-drying process in a Labconco freeze dryer. (b) Absorptance spectra of the $2 \mathrm{wt} \% \mathrm{GG} / \mathrm{CI}$ hydrogel with randomly porous structures and with vertical channels, respectively. (c) Thermal conductivity of the GG/CI hydrogel with vertical channels fabricated with different GG concentrations (1, 2, and $3 \mathrm{wt} \%)$ and with porous structures fabricated with $2 \mathrm{wt} \%$ GG solution $(2(\mathrm{P}))$ at the dry state and wet state, respectively. (d) Saturation water absorption capacity of the GG/CI hydrogel evaporator with vertical channels fabricated with different GG concentrations (1, 2, and 3) and with porous structures fabricated with $2 \mathrm{wt} \%$ GG solution (2(P)). (e) Central surface temperature evolutions of the $2 \mathrm{wt} \% \mathrm{GG} / \mathrm{CI}$ hydrogels with vertical channels and with randomly porous structure under one sun illumination within $1 \mathrm{~h}$. (f) Mass changes of water for GG/CI hydrogel evaporators with vertical channels and with porous structures, respectively, under one sun. 

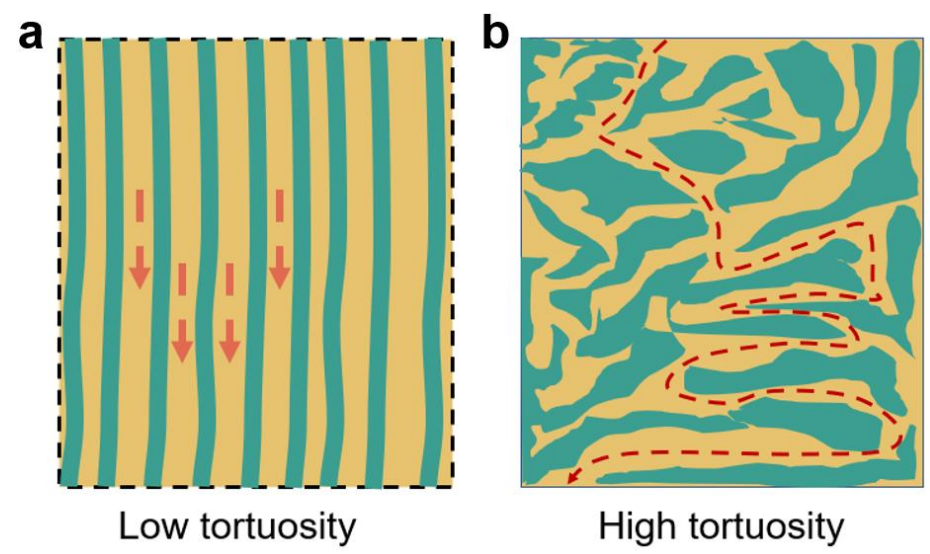

Figure S15. Schematic illustrating the salt rejection process of the GG/CI hydrogel with (a) vertical microchannels and (b) random porous structures. 

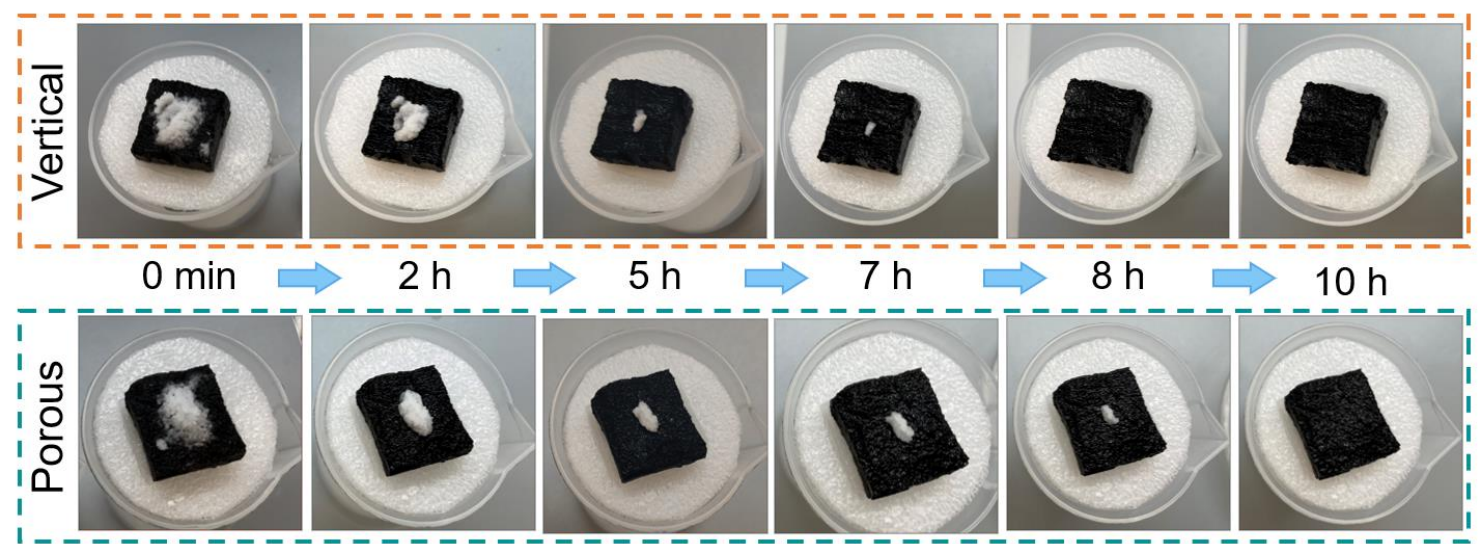

Figure S16. Time-lapse images showing the self-cleaning property difference of the GG/CI hydrogel evaporator with vertical microchannels and random porous structures. 